\title{
Prediction of phase ambiguity resolution based on signal intensity and geometry
}

\author{
Daniel Willi $\cdot$ Jan Skaloud
}

Received: 10 March 2014/ Accepted: 1 September 2014/Published online: 23 September 2014

(C) Springer-Verlag Berlin Heidelberg 2014

\begin{abstract}
The goal of this contribution was to develop a reliable quality indicator reflecting the potential success of differential carrier phase ambiguity resolution for kinematic Global Positioning System (GPS). The indicator must be operable without any communication link between the rover and the reference station. Two common existing GPS quality measures are combined: firstly, the SIGMA model, which is a signal intensity-based weighing scheme reflecting the actual signal quality, and secondly, the ambiguity dilution of precision (ADOP), which is an indicator of the geometric strength of a constellation. Together, these two established indicators form the newly developed ADOP+. In the first part of the present work, a calibration is conducted in order to obtain the parameters for the SIGMA model. In the second part, the $\mathrm{ADOP}+$ is derived and implemented. A practical test is performed on a motorcycle in order to validate the concept. The $\mathrm{ADOP}+$ is proven to be meaningful and well performing within the empirical case. It is able to predict the success of phase ambiguity resolution in the majority of situations.
\end{abstract}

Keywords Kinematic GNSS - Ambiguity resolution · Ambiguity dilution of precision

\section{Introduction}

In kinematic global positioning system (GPS) applications, centimeter- to decimeter-level positioning accuracy can

\section{Willi $(\bowtie) \cdot$ J. Skaloud}

Geodetic Engineering Laboratory, Swiss Federal Institute of

Technology Lausanne, EPFL ENAC TOPO, Bâtiment GC,

Station 18, 1015 Lausanne, Switzerland

e-mail: daniel.willi@gmail.com

J. Skaloud

e-mail: jan.skaloud@epfl.ch only be achieved by solving phase ambiguities in differential mode (Kaplan and Hegarty 2006). Without a communication link between the rover and the reference receiver, the success of ambiguity resolution cannot be checked in real time. This can be problematic for some airborne surveying, as in airborne photogrammetry with unmanned aerial vehicles (UAV). Possible tilt of the antenna and intentional or unintentional signal interferences are one possible difficulty, others are related to signal reception shading by natural or artificial structures (mountains, buildings, etc.). A reliable real-time quality check allows in situ assessment of the collected data, helps to avoid additional field work and reduces the costs of data acquisition.

The success of kinematic ambiguity resolution depends mainly on the actual signal quality and on the geometry and the redundancy of the satellite constellation. The goal of this contribution is to develop a quality indicator, applicable in real time and without a communication link to the reference station, which takes into account both aspects, the signal quality as well as the receiver to satellite geometry. The concept should be as general as possible and rely on a strict mathematical definition.

\section{Concept}

The newly developed quality indicator, the ADOP+, is a combination between a signal intensity-based weighting scheme of code and phase measurements, the SIGMA- $\varepsilon$ model (Brunner et al. 1999), and a geometrical quality indicator (Teunissen and Odijk 1997), the ambiguity dilution of precision (ADOP). The stochastic SIGMA- $\varepsilon$ model establishes a link between the signal intensity and the variance of code or phase observations. Hence, it is able to account for signal jamming and diffraction. The stochastic 
model is used as input for the ADOP+ computation, which is enhanced thanks to realistic weights for each observation. Since the ambiguity search space and therefore the $\mathrm{ADOP}+$ value are mainly dependent on the estimated accuracy of the code solution, a correct weighting of the code observations, through the SIGMA model, is essential.

In the first part of this contribution, the selected stochastic model and its realization are described. A calibration is conducted to derive the parameters of the model. The second part addresses the derivation and the implementation of the ADOP + . Practical tests are carried out to validate the presented concept.

\section{Stochastic modeling}

A stochastic model describes the probabilistic behavior of observations. In the present case, the accuracy of code and phase measurements is estimated based on the intensity of the received GPS signal. A low GPS signal intensity indicates perturbations and therefore a high noise level in the measurements. The aim of this section is to derive and to calibrate a parameterized model for the measurement accuracy.

\section{Measuring signal intensity}

Signal intensity is reflected either by the signal-to-noise ratio (SNR) or by the carrier-to-noise power-density ratio $C / N_{0}$. The SNR is defined as the ratio of the signal power, usually the carrier power, to the noise power in a given bandwidth. It is expressed in decibels. In contrast, the $C / N_{0}$ is defined as the ratio of the carrier power and the noise power per unit of bandwidth. Usually, this measure is expressed in decibel-hertz. The $C / N_{0}$ can be measured in each tracking loop, but usually, only one $C / N_{0}$ value per carrier frequency is available in the receiver output.

\section{Variance of observations}

A link between the signal intensity and the variance of a GPS code or phase observation can be established as follows (Kaplan and Hegarty 2006).

$\sigma_{\mathrm{c}}^{2}=\frac{\alpha B_{\mathrm{c}}}{c / n_{0}}\left(1+\frac{2}{T c / n_{0}}\right) \lambda_{\mathrm{c}}^{2}$

$\sigma_{\mathrm{p}}^{2}=\frac{B_{\mathrm{p}}}{c / n_{0}}\left(1+\frac{1}{2 T c / n_{0}}\right) \frac{\lambda^{2}}{4 \pi^{2}}$

$\sigma_{\mathrm{c}}^{2}\left[\mathrm{~m}^{2}\right]$ is the variance of an undifferentiated code observation, $\sigma_{\mathrm{p}}^{2}\left[\mathrm{~m}^{2}\right]$ is the variance of an undifferentiated phase observation, $\alpha[-]$ is the code tracking loop discriminator correlator factor (either 1 for a shared correlator or 0.5 for dedicated early and late correlators), $B_{\mathrm{c}}[\mathrm{Hz}]$ and
$B_{\mathrm{p}}[\mathrm{Hz}]$ are the bandwidths of the corresponding tracking loop, $\lambda_{\mathrm{c}}[\mathrm{m}]$ is the code wavelength, $\lambda[\mathrm{m}]$ is the carrier wavelength, and $T[\mathrm{~s}]$ is the predetection integration time. $c / n_{0}[\mathrm{~Hz}]$ is the $C / N_{0}[\mathrm{~dB}-\mathrm{Hz}]$ value expressed as a ratio:

$c / n_{0}=10^{\frac{C / N_{0}}{10}}$

The second term in brackets in Eqs. 1 and 2 is called squaring loss. It reflects the nonlinear behavior of the measurement accuracy in case of low signal intensities.

\section{The SIGMA model}

Based on Eqs. 1 and 2, the SIGMA- $\varepsilon$ model (Brunner et al. 1999; Wieser and Brunner 2000) establishes a parametric relation between the signal intensity and the variance of an undifferentiated observation:

$\sigma_{i}^{2}=V_{i}+C_{i} \cdot 10^{\frac{-C / N_{0}}{10}}$

$\sigma_{i}^{2}\left[\mathrm{~m}^{2}\right]$ is the variance of an undifferentiated observation. $V_{i}\left[\mathrm{~m}^{2}\right]$ and $C_{i}\left[\mathrm{~m}^{2} \mathrm{~Hz}\right]$ are two parameters and $C / N_{0}$ [dB-Hz] is the carrier-to-noise power-density ratio. The subscript $i$ indicates the type of observation, for instance, C/A code on L1 or carrier phase measurements on L2. Compared with Eqs. 1 and 2, the squaring loss is neglected and the remaining parameters are combined to a unique parameter $C_{i}$. Furthermore, an offset $V_{i}$ is added to the equation. Brunner et al. (1999) suggest to determine $V_{i}$ and $C_{i}$ in a calibration procedure. Since the variance of a measurement depends on the bandwidth of the corresponding tracking loop, a calibration has to be conducted for each receiver type. Due to the dependence on the wavelength, a set of parameters must be determined for each observation type. Additionally, the parameters of the SIGMA model were found to be dependent on the antenna type.

\section{Calibration procedure}

In order to eliminate various errors, such as orbit errors, synchronization errors, or the influence of the troposphere, the calibration of the model shown in Eq. 4 has to be conducted on double-difference level. On this level, the parameters of the SIGMA model do not appear explicitly anymore. As suggested by the authors of the model, the SIGMA variance can be estimated without any parameter (see Eq. 4):

$\sigma_{i}^{2}=10^{\frac{-C / N_{0}}{10}}$

Once this variance is computed for each observation, variance propagation can be applied in order to obtain the estimated SIGMA variance of the double differences (see Eqs. 16-18). The estimated SIGMA variance can then be used to classify the double differences. Each class regroups 
$p$ double differences which are expected to have similar precisions. Assuming a perfectly known baseline, the empirical variance $\sigma_{\mathrm{emp}}^{2}$ of each class of double differences can be computed:

$\sigma_{\text {emp }}^{2}=\frac{1}{p-1} \mathbf{v}^{\mathrm{T}} \mathbf{v}$

$\mathbf{v}$ is the vector of the double-difference residuals. At this stage, the model parameters are estimated with a linear regression between the SIGMA variance, derived from Eq. 5 and propagated to the double-difference level, and the actual empirical variance from Eq. 6. An example is shown in Fig. 1. The intercept corresponds to $V_{i}$ and the slope of the regression to $C_{i}$ (see Eq. 4). The quality of the regression can be quantified by the coefficient of determination $R^{2}$, which is defined as follows.

$R^{2}=1-\frac{\sum_{i=1}^{n}\left(Y_{i}-\hat{Y}_{i}\right)^{2}}{\sum_{i=1}^{n}\left(Y_{i}-\bar{Y}_{i}\right)^{2}}$

$Y_{i}$ is the ordinate, $\hat{Y}_{i}$ is its estimate and $\bar{Y}_{i}$ is the mean value. A coefficient value of $R=1$ indicates that the whole variance of the dataset is perfectly reflected by the linear regression.

\section{Practical realization}

As a basis for the calibration, static measurements were collected over $24 \mathrm{~h}$ in a zero-baseline setup. Two Javad receivers equipped with OEM-boards TR-62T (L1/L2/L5, Galileo, SBAS) and TR-63T (L1/L2/L5, GLONASS, Galileo, SBAS), and four different GPS antennas were used (Table 1). The dynamic adaptation of the tracking loops

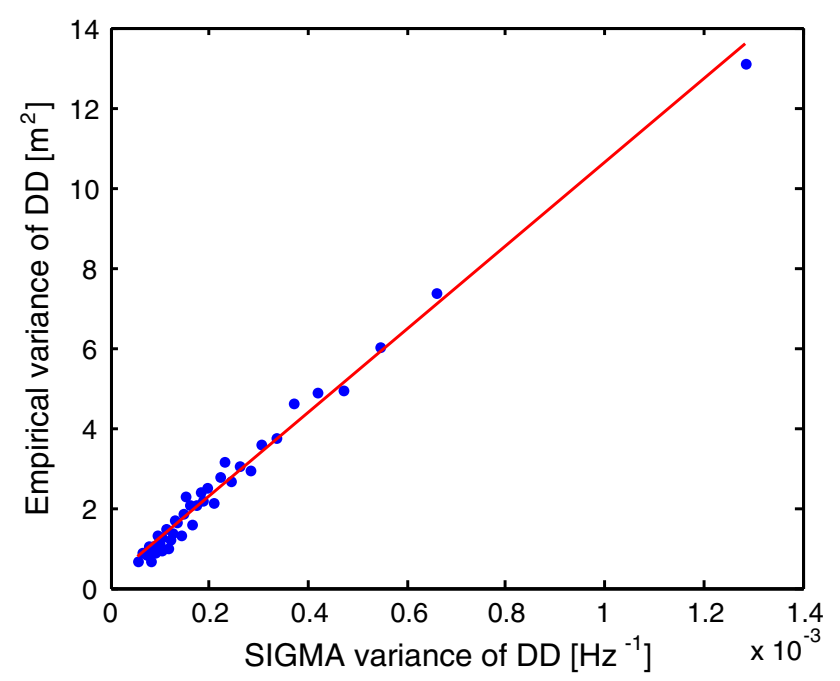

Fig. 1 Calibration of the SIGMA model parameters via linear regression for $\mathrm{C} / \mathrm{A}$ code measurements with the Antcom G5 antenna. The slope of the regression is equal to $\mathrm{C}$, its offset to $\mathrm{V}$
Table 1 Calibrated antennas

\begin{tabular}{|c|c|c|c|c|}
\hline & Brand & Type & Remark & $\begin{array}{l}\text { Weight } \\
(\mathrm{kg})\end{array}$ \\
\hline 1 & Antcom & G5Ant-1AS1 & $\mathrm{L} 1 / \mathrm{L} 2$ + GLONASS & 0.100 \\
\hline 2 & Maxtena & $\begin{array}{l}\text { M1227HCT- } \\
\text { A-SMA }\end{array}$ & L1/L2 + GLONASS & 0.017 \\
\hline 3 & Topcon & G3-A1 & $\begin{array}{l}\text { Geodetic antenna, L1/L2/ } \\
\text { L5 + GLONASS }\end{array}$ & 0.550 \\
\hline 4 & Unknown & MK-76 & L1 only & 0.020 \\
\hline
\end{tabular}

bandwidths by the receiver was disabled, so that the parameters $V_{i}$ and $C_{i}$ can be considered as constants. A set of these parameters is determined for the following observation types: C/A code, P2 code, L1 phase, and L2 phase.

\section{Validation}

In order to check the robustness of the model, datasets were acquired in two operational-like environments: firstly, with a tilted antenna and secondly, on a static UAV. In the first case, the antenna was tilted by $40^{\circ}$ to increase the measured ground noise and to rotate the antenna gain pattern with respect to zenith. In the second case, all on-board systems on the UAV, engines, communication devices, and the inertial navigation unit, were activated to simulate unintentional interferences. The parameters of the SIGMA model, $V_{i}$ and $C_{i}$, determined previously, are applied to these datasets. The same procedure as for the calibration is used, but instead of performing a linear regression, the accordance of the existing parameters with the new dataset is checked through the coefficient of determination $R^{2}$.

\section{Results}

The calibration results for the code measurements with the Antcom G5 antenna are shown in Table 2. The model performs well in the optimal environment: The coefficient of determination reaches $98 \%$. The coefficients of determination are lower for the validations, which were expected since the parameters were fit to the first dataset. $R^{2}$ exceeds $80 \%$ in the inclined environment, confirming the validity and the robustness of the model. The value drops to $67 \%$ for the validation on the UAV. This is partially caused by the short observation time. For security reasons, the running UAV had to be under permanent supervision. Therefore, the measurement duration does not exceed $3 \mathrm{~h}$. The results for the phase measurements are more delicate to interpret. The linear regression leads to an overestimation of $V_{i}$ and an underestimation of $C_{i}$. For that reason, a second regression with $V_{i}$ forced to zero was conducted (Fig. 2). The calibration results for the Topcon 
Table 2 Calibration and validation results for C/A code measurements with the Antcom G5 antenna

\begin{tabular}{llllll}
\hline & Environment & $\begin{array}{l}\text { Mean } \\
\mathrm{R}^{2}(\%)\end{array}$ & $\begin{array}{l}\mathrm{C} \\
{\left[\mathrm{m}^{2} \mathrm{~Hz}\right]}\end{array}$ & $\begin{array}{l}\mathrm{V} \\
{\left[\mathrm{m}^{2}\right]}\end{array}$ & $\begin{array}{l}\text { Data } \\
\text { duration (h) }\end{array}$ \\
\hline Calibration & Optimal & 98.3 & 10,758 & 0.157 & 24 \\
Validation & Inclined $40^{\circ}$ & 82.1 & & & 24 \\
Validation & UAV & 67.0 & & & 3 \\
\hline
\end{tabular}

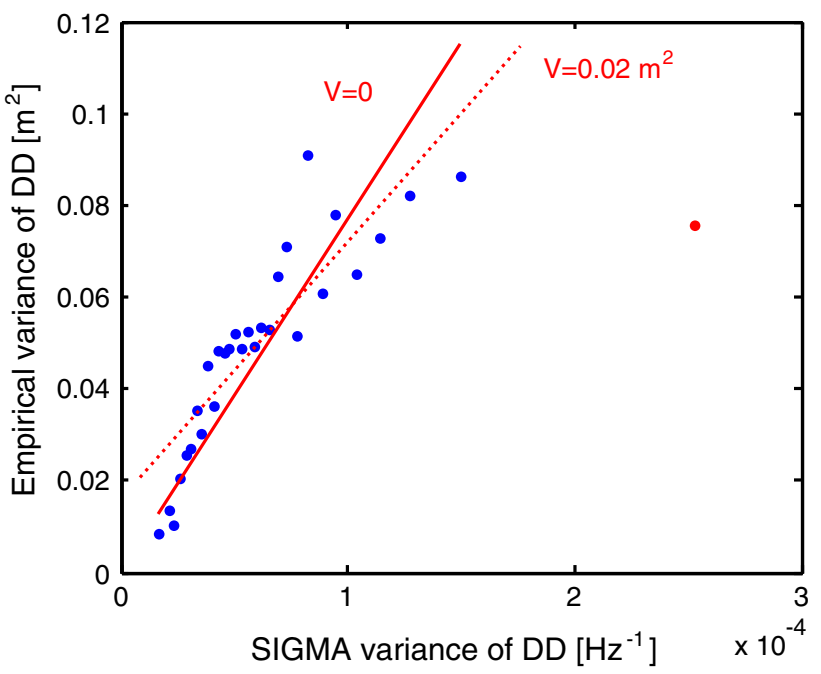

Fig. 2 Linear regression for phase measurements with the Topcon antenna. The dotted line shows the linear regression without any condition, the solid line with $\mathrm{V}$ forced to zero. The red dot is an outlier

geodetic antenna are shown in Table 3. In general, the coefficients of determination reach lower values for the phase measurements than for the code observations. Enhancements in cycle slip detection and phase ambiguity resolution, which are needed to compute the correct variance of the phase measurements, could help to improve these results.

\section{Discussion}

The SIGMA model could be implemented successfully. A validation in various environments showed that the model is robust with respect to changes in the environment. The model performances for code measurements are extremely high with coefficients of determination up to $98 \%$. The model was also calibrated for carrier phase observations. The coefficient of determination decreases below $70 \%$ and therefore indicates a lower agreement between the model and the data. As a direct result of this calibration, the parameters of the SIGMA- $\varepsilon$ model are obtained for various antennas and various observation types, namely $\mathrm{C} / \mathrm{A}$ code, P2 code, L1 phase, and L2 phase.
Table 3 Results of the calibration for phase measurements with the Topcon antenna

\begin{tabular}{llll}
\hline & Mean $\mathrm{R}^{2}(\%)$ & $\mathrm{C}\left[\mathrm{m}^{2} \mathrm{~Hz}\right]$ & $\mathrm{V}\left[\mathrm{m}^{2}\right]$ \\
\hline V as parameter & 69.3 & 462 & 0.020 \\
V forced to zero & 64.9 & 736 & 0 \\
\hline
\end{tabular}

\section{ADOP}

The dilution of precision (DOP), which is a kind of prior analysis, is a popular concept in standalone GPS (Kaplan and Hegarty 2006). The computation of this single-value indicator does not require any measurements and relies only on a stochastic and a functional model. The DOP reflects the geometrical strength of a constellation. If the functional model is adapted and extended by an additional parameter, the phase ambiguity, the ADOP is obtained (Teunissen 1997). In the following, the derivation of the traditional ADOP is shown, as well as its combination with the stochastic model from the previous section, resulting in the new ADOP+. Finally, the performance of the ADOP+ is compared to the classical, purely geometrical ADOP.

\section{Definition}

The ADOP is defined as following (Teunissen and Odijk 1997)

$\mathrm{ADOP}=\left(\operatorname{det} \mathbf{Q}_{\hat{a} \hat{a}}\right)^{\frac{1}{2 n}}$

$\mathbf{Q}_{\hat{a} \hat{a}}$ is a sub matrix of the parameter cofactor matrix $\mathbf{Q}_{\hat{x} \hat{x}}$ and contains the cofactors of the ambiguities. $\mathbf{Q}_{\hat{x} \hat{x}}$ is well known from adjustment theory as:

$\mathbf{Q}_{\hat{x} \hat{x}}=\left(\mathbf{A}^{\mathrm{T}} \mathbf{Q}_{l l}^{-1} \mathbf{A}\right)=\mathbf{N}^{-1}$

$\mathbf{Q}_{l l}$ is the cofactor matrix of the observations. $\mathbf{N}$ is called the normal equation matrix. $\mathbf{A}$ is the matrix of partial derivatives of the function $\mathbf{f}(\mathbf{x})$ with respect to the parameters $\mathbf{x}$. $\mathbf{f}(\mathbf{x})$ represents the functional model and establishes the link between true parameters $\mathbf{x}$ (e.g., coordinates) and true observations $\mathbf{l}$ (e.g., code and phase observations):

$\mathbf{l}=\mathbf{f}(\mathbf{x})$

By using the determinant and not only the trace of $\mathbf{Q}_{\hat{a} \hat{a}}$, the correlation between ambiguities is taken into account by the ADOP.

Properties

The value of the ADOP is dependent on the number of satellites and, if a geometry-based functional model is chosen, dependent on the satellite constellation. Furthermore, the 
ADOP depends on the relative and absolute weights attributed to each observation. The effect, on the ADOP, of the accumulation of epochs in a sequential adjustment is as follows (Willi 2014).

$\mathrm{ADOP} \propto\left(\frac{1}{k}\right)^{1 / 2}$

This relation is valid, assuming that the $k$ epochs are independent and geometrically identical with constant weights.

\section{Functional model}

A geometry-based model with observations on two frequencies is used. In a simplified notation and for a single double difference, the model reads as follows (Teunissen 1997).

$$
\begin{aligned}
& \nabla \Delta P_{1}=\nabla \Delta \rho \\
& \nabla \Delta L_{1}=\nabla \Delta \rho+\lambda_{1} \nabla \Delta N_{1} \\
& \nabla \Delta P_{2}=\nabla \Delta \rho \\
& \nabla \Delta L_{2}=\nabla \Delta \rho+\lambda_{2} \nabla \Delta N_{2}
\end{aligned}
$$

$\Delta \nabla P$ is a double-difference code observation, and $\Delta \nabla L$ is a double-difference carrier phase observation with its double-difference ambiguity $\Delta \nabla N . \Delta \nabla \rho$ is the geometric range on double-difference level, and $\lambda_{1}$ is the wavelength of the first, $\lambda_{2}$ the wavelength of the second GPS frequency. After linearization, the model has the following shape:

$$
\left(\begin{array}{c}
\Delta \nabla P_{1} \\
\Delta \nabla L_{1} \\
\Delta \nabla P_{2} \\
\Delta \nabla L_{2}
\end{array}\right)=\mathbf{A} \cdot \delta \mathbf{x}=\mathbf{A} \cdot\left(\begin{array}{c}
X \\
Y \\
Z \\
\Delta \nabla N_{1} \\
\Delta \nabla N_{2}
\end{array}\right)
$$

If, in a kinematic case, observations are taken over several epochs, a set of unknown coordinates must be added to the vector of parameters for each epoch. In contrast, the ambiguity terms stay constant, assuming the absence of cycle slips. The redundancy of the model can be read from Table 4.

\section{Stochastic model}

In the absence of any information, the observations are assumed to have uniform weights. Usually, the ratio between the variance of a code observation $\sigma_{\mathrm{c}}^{2}$ and the variance of a phase observation $\sigma_{\mathrm{p}}^{2}$ is set to:

$\frac{\sigma_{\mathrm{p}}^{2}}{\sigma_{\mathrm{c}}^{2}}=10^{-4}$

\section{Stochastic Model of the ADOP+}

The ADOP + is a combination between the traditional ADOP and a realistic stochastic model. The variances of the observations at the rover $\mathbf{K}_{l l, A}$ are estimated with the SIGMA model (see previous section), and the variances of the observations at the reference station $\mathbf{K}_{l l, B}$ are estimated with an elevation-dependent model. The reason for this choice is simple: At the rover, no data from the reference station are available. Therefore, it is not possible to compute the SIGMA variances for the reference station. The elevation-dependent model works very well in clear sky condition, which is assumed to be the case at the reference station. The elevation-dependent model reads as follows.

$\sigma_{j, B}^{2}=U_{i}+B_{i} \frac{1}{\sin E l_{j}}$

$E l_{j}$ is the elevation of the $j^{\text {th }}$ satellite. The subscript $i$ stands for the observation type, for instance, C/A code or L2 phase. To simplify the computation of the elevation, it is assumed that the elevation of the satellite seen from the rover is equal to the elevation of the satellite seen from the reference station. The parameters $U_{i}$ and $B_{i}$ were determined within the SIGMA model calibration procedure.

In the second step, classical variance propagation (or error propagation) is applied in order to obtain the variances at double-difference level $\mathbf{K}_{\nabla \Delta}$. Both the variances from the rover $\mathbf{K}_{l l, A}$ and the variances from the reference $\mathbf{K}_{l l, B}$ contribute to the error budget of the double differences:

$\mathbf{K}_{\nabla \Delta}=\mathbf{D} \cdot\left(\mathbf{K}_{l l, A}+\mathbf{K}_{l l, B}\right) \cdot \mathbf{D}^{\mathrm{T}}$

D is the sequential differentiation operator, which is used to form the double differences out of the single differences:

$$
\begin{aligned}
\mathbf{D}_{m(n-1) \times m \cdot n}= & \left(\begin{array}{ll}
\boldsymbol{I}_{m(n-1)} & 0_{m(n-1), m}
\end{array}\right) \\
& -\left(\begin{array}{ll}
0_{m(n-1), m} & \boldsymbol{I}_{m(n-1)}
\end{array}\right)
\end{aligned}
$$

$m$ is the number of observations per satellite, and $n$ is the number of satellites. Additionally, $\sigma_{0}$ was chosen to be one, so that:
Table 4 Overdetermination of the geometry-based functional model

\begin{tabular}{lllll}
\hline Model & Mode & Unknowns & Observations & Overdetermination \\
\hline \multirow{2}{*}{ Geometry based double frequency } & Kinematic & $3 k+2 n-2$ & $4 k(n-1)$ & $k(4 n-7)-2 n+2$ \\
& Static & $2 n+1$ & $4 k(n-1)$ & $4 k(n-1)-2 n-1$ \\
\hline
\end{tabular}




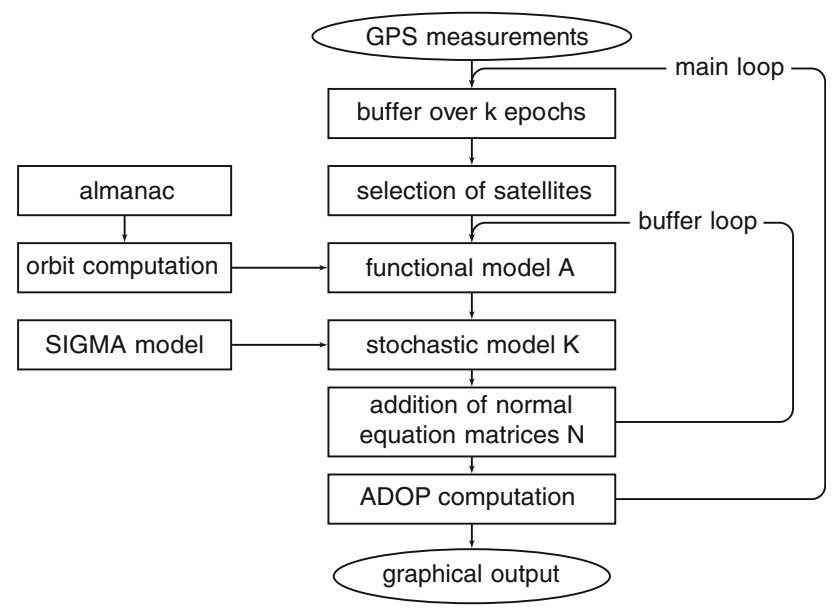

Fig. 3 Structure of the ADOP+ implementation

$\mathbf{K}_{\nabla \Delta}=\sigma_{0}^{2} \mathbf{Q}_{\nabla \Delta}=\mathbf{Q}_{\nabla \Delta}$

Implementation

The implementation of the ADOP+ is diagrammed in Fig. 3. The program enters the slow loop. A buffer is formed, containing all satellites measured over the buffer length of $k$ epochs. $k$ has to be chosen according to the rover dynamics and the sampling rate. On a highly dynamic vehicle, a buffer length of a few seconds is reasonable. The satellites presenting a data outage within the buffer are eliminated. Since satellites need to have been tracked without interruption on both frequencies, this operation is implicitly a cycle slip detection through loss of tracking on L2. After the satellite selection, the program enters the fast loop and the functional model $\mathbf{A}$ is formed, using satellite orbits computed from the ephemerides. The SIGMA model is used to set up the stochastic model $\mathbf{K}$. Finally, the normal equation matrix $\mathbf{N}$ is formed. This operation is repeated for all $k$ epochs contained in the buffer, and the normal equation matrices are summed up in order to perform a sequential adjustment. Once the program quits the fast loop, the normal equation matrix is inverted and the ADOP+ is extracted. The buffer is shifted, and the ADOP+ is computed for the following epochs.

\section{Validation}

A validation was conducted on a motorcycle. This vehicle was chosen mainly for practical reasons: it presents interesting dynamics, a changing inclination in turns, and it can be deployed without any special authorization. Figure 4 shows the motorcycle with the Maxtena antenna mounted on a pole at the back. Several short baseline $(<12 \mathrm{~km})$ tests were performed in challenging urban and suburban environments. The Topcon antenna on the roof of an EPFL

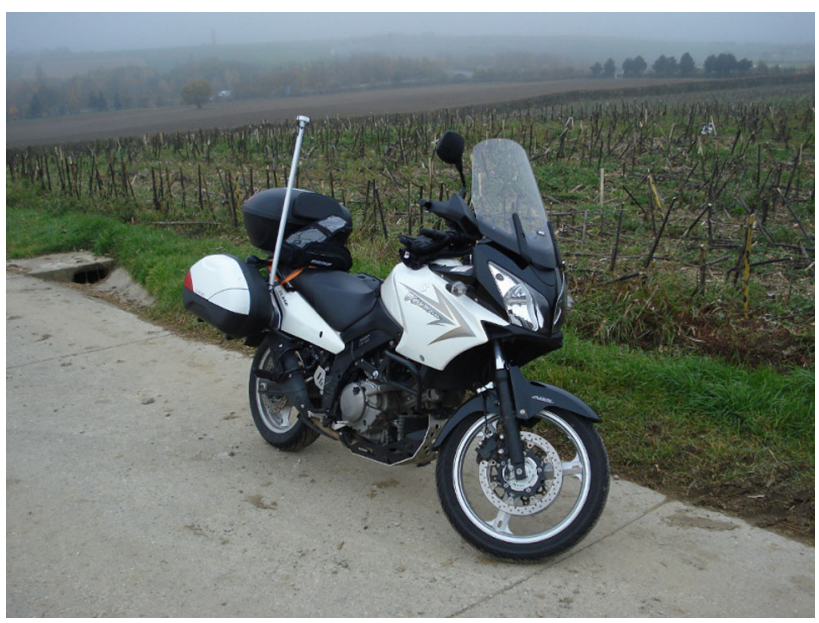

Fig. 4 Empirical setup on a Suzuki DL650 V-Strom with the Antcom $G 5$ on a pole at the back

building connected to a Javad multi-frequency receiver served as reference station. The same types of receivers were used as within the calibration. The baselines are computed in postprocessing with the commercial software GrafNav (http://www.novatel.com). As this software is proprietary, the exact algorithm on ambiguity resolution and the criteria on ambiguity validation are not known; nevertheless, it is considered by practitioners as a standard for its proven performance, reliability, and versatility in kinematic applications. In the last step, the phase ambiguity fixing rate in postprocessing is compared to the ADOP prediction.

\section{Results and discussion}

Figure 5 shows the results of the validation in the suburban environment. The dataset was acquired with a measuring rate of $10 \mathrm{~Hz}$. The buffer length is set to $k=50$ epochs or $5 \mathrm{~s}$. The black line shows the prediction computed with the $\mathrm{ADOP}+$. The background is the graphical output from the commercial software: green indicates successful ambiguity fixing in the forward and backward solutions, blue indicates an ambiguity resolution in only one direction, and red represents an unsuccessful ambiguity resolution. The implemented ADOP+ is able to predict the problematic periods (1) and (2). The Sects. (3) and (4) are also correctly predicted, but with lower ADOP values. The episodes with unproblematic ambiguity resolution are also correctly detected, like (5) and (6). In some cases, the $\mathrm{ADOP}+$ turns out to be over pessimistic (7). In general, the ADOP+ is able to successfully predict the achievement of ambiguity resolution. Setting an alarm threshold between 0.6 and 0.8 cycles seems a reasonable choice. In general, the threshold has to be chosen based on empirical tests and depending on the required ambiguity fixing rate. 


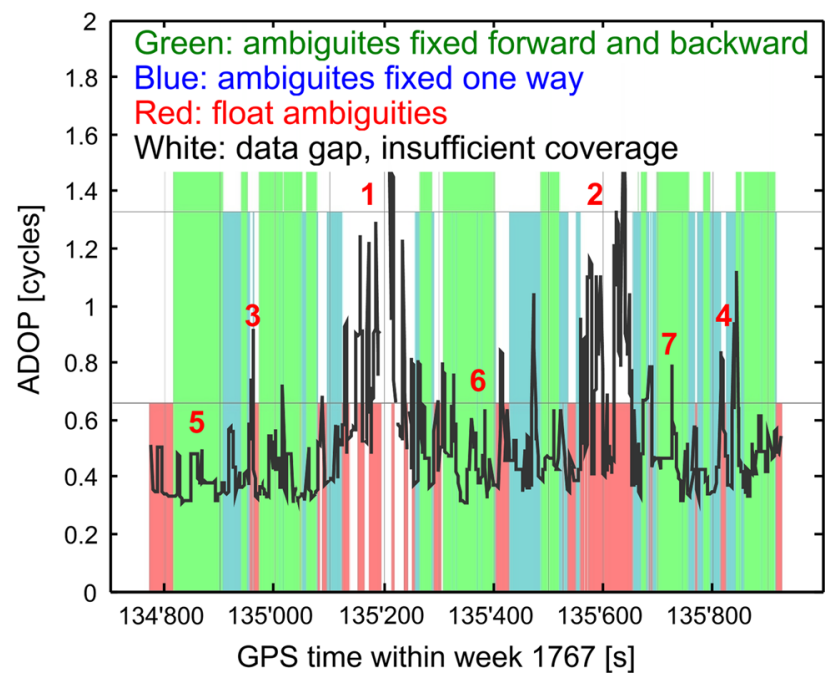

Fig. 5 Result of the prediction with the ADOP+ in a suburban environment. The buffer length is equal to 50 epochs, the measurement rate to $10 \mathrm{~Hz}$

In the case that the buffer length $k$ is modified, Eq. 11 has to be used in order to properly scale the resulting ADOP+ values.

Figure 6 illustrates the benefit of using a signal intensity-based weighting. The upper line represents the newly developed $\mathrm{ADOP}+$, including stochastic input from the SIGMA model and the elevation-dependent model. The lower line shows the traditional ADOP computed with fixed weights. In this case, the signal intensity values do not enter the computation and the ADOP only depends on the geometry and the redundancy of the satellite constellation. This fixed and unitary weighting introduces an arbitrary scale; hence, only the relative values of the ADOP and the ADOP+ can be compared and not their absolute values. In cases of a favorable satellite constellation and a good signal quality, the traditional ADOP works well, e.g., (1), (2), and (3). In this case, there is only a little benefit using a signal intensity-based stochastic model as input. However, as soon as the signal quality gets worse, only the ADOP+ (upper line) is able to correctly predict difficulties in phase ambiguity resolution, as in (4) to (7). In some cases, for instance, in (5) and (7), the traditional ADOP (lower line) shows no or only a little peak and overestimates the success of phase ambiguity resolution. In other cases, the traditional ADOP shows some peaks, but drops to small values in between.

In summary, the traditional ADOP is unable to detect difficulties in ambiguity fixing that occur despite a strong satellite constellation. In contrast, the stochastic SIGMA model input enhances the sensitivity and the performance of the ADOP+. Since the computational cost of the

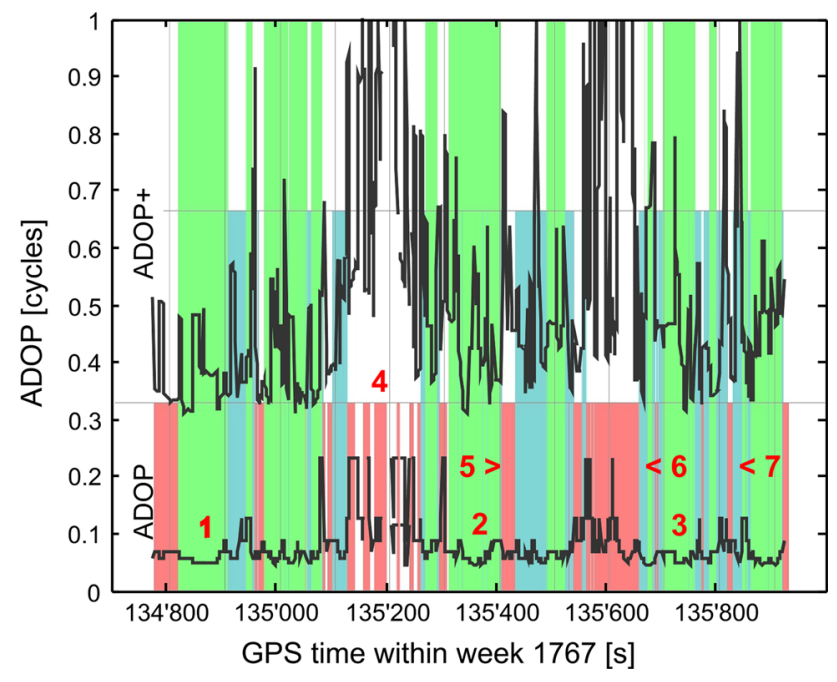

Fig. 6 Comparison of the ADOP+ (upper line) and the traditional, purely geometrical ADOP (lower line)

SIGMA model is small compared to the matrix inversion operations, the ADOP+ is well suited to predict the phase ambiguity resolution success rate directly on a rover.

\section{Conclusions}

In the first part, a calibration has successfully been conducted in order to obtain the parameters of the stochastic SIGMA model. The calibration gives good results for the estimation of the accuracy of code measurements, which have the largest impact on the ADOP+ values. Parameters for other types of observations could be obtained, but the residuals are higher. In the second part, an ambiguity resolution success prediction was implemented. It could be shown that the newly developed indicator, the ADOP+, is able to correctly predict the success rate of ambiguity resolution in the majority of cases, thanks to its enhancement by realistic weights from the SIGMA model.

\section{Outlook}

The concept of the ADOP+ is proven to work and to be meaningful in the context of kinematic differential carrier phase ambiguity resolution. Practical tests with this indicator revealed its whole potential. The possible benefits are manifold, from applications in photogrammetry to land surveying. Further studies have to be conducted in order to properly take into account the correlation between observations in the case of high frequency measurements. Another improvement could be the integration of the baseline length in the functional model through an 
observation of the ionospheric delay, resulting in a better modeling of long baselines $(>5 \mathrm{~km})$.

Acknowledgments We would like to thank Prof. Bertrand Merminod from the Geodetic Engineering Lab at the Swiss Federal Institute of Technology Lausanne (EPFL) for hosting and co-supervising and Prof. Dr. Alain Geiger from the Geodesy and Geodynamics Lab at the Swiss Federal Institute of Technology Zurich (ETH) for cosupervising the present master thesis.

\section{References}

Brunner FK, Hartinger H, Troyer L (1999) GPS signal diffraction modelling: the stochastic SIGMA- $\Delta$ model. J Geod 73(5):259-267. doi:10.1007/s001900050242

Kaplan ED, Hegarty CJ (eds) (2006) Understanding GPS: principles and applications. Artech House, Boston

Teunissen PJG (1997) Precision, volume and eigenspectra for GPS ambiguity estimation based on the time-averaged satellite geometry. J Geod 71(5):290-301. doi:10.1007/s001900050097

Teunissen PJG, Odijk D (1997) Ambiguity Dilution of Precision: definition, properties and application. Proc. ION-GPS-1997, Institute of Navigation, 16-19 Sept, Kansas City, MO, USA, pp 891-899

Wieser A, Brunner FK (2000) An extended weight model for GPS phase observations. Earth Planets Space 52(10):777-782

Willi D (2014) Prédiction de la résolution des ambiguïtés de phase en fonction de l'intensité du signal et de la géométrie. Master thesis, Swiss Federal Institute of Technology, Lausanne, Switzerland

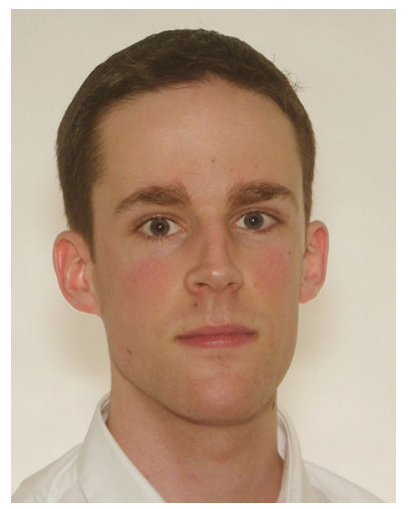

Daniel Willi obtained a M.Sc. degree in geomatics from the Swiss Federal Institute of Technology Zurich (ETH) in 2014. His main fields of interest are engineering geodesy and satellite geodesy. He holds a B.Sc. degree in environmental engineering from the Swiss Federal Institute of Technology Lausanne (EPFL).

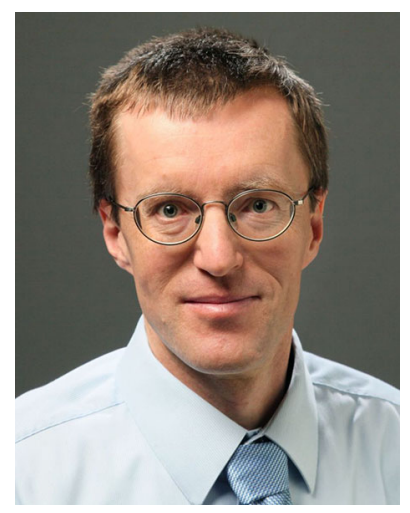

Jan Skaloud is a lecturer and senior scientist at the Swiss Federal Institute of Technology Lausanne (EPFL). He holds Ph.D. and M.Sc. degrees in Geomatics Engineering from Canada (The University of Calgary), and Dipl. Ing. degree from CVUT (Prague, Czech Rep.). He is the past and current Chair of working groups of the International Association of Geodesy (IAG) and International Society for Photogrammetry and Remote Sensing (ISPRS). In 2009, he was recognized by the journal GPS World as one of the 50 world's most influential scientists in the field of satellite navigation. 